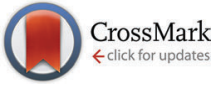

Cite this: Phys. Chem. Chem. Phys., $2014,16,25404$

Received 19th September 2014, Accepted 17th October 2014

DOI: $10.1039 / c 4 c p 04217 d$

www.rsc.org/pccp

\section{Selective nucleation of iron phthalocyanine crystals on micro-structured copper iodide}

\author{
Luke A. Rochford, ${ }^{\star a}$ Alexandra J. Ramadan, ${ }^{b}$ Sandrine Heutz ${ }^{b}$ and Tim S. Jones ${ }^{a}$ \\ Morphological and structural control of organic semiconductors through structural templating is an \\ efficient route by which to tune their physical properties. The preparation and characterisation of iron \\ phthalocyanine (FePc)-copper iodide (Cul) bilayers at elevated substrate temperatures is presented. Thin \\ Cul(111) layers are prepared which are composed of isolated islands rather than continuous films \\ previously employed in device structures. Nucleation in the early stages of FePc growth is observed at \\ the edges of islands rather than on the top (111) faces with the use of field emission scanning electron \\ microscopy (FE-SEM). Structural measurements show two distinct polymorphs of FePc, with Cul islands \\ edges nucleating high aspect ratio FePc crystallites with modified intermolecular spacing. By combining \\ high substrate temperature growth and micro-structuring of the templating Cul(111) layer structural and \\ morphological control of the organic film is demonstrated.
}

\section{Introduction}

Control of morphology and crystal structure in organic semiconductor films affords the ability to tune their physical and electronic properties for specific device applications. ${ }^{1-3}$ Inorganic thin films have been widely employed as structural modification layers for the improvement of device parameters. ${ }^{4,5}$ A significant example of this methodology is the insertion of an evaporated copper(I) iodide layer to alter the structure of a planar metallophthalocyanine (MPc) epilayer. ${ }^{6}$ The effect of such a structural modification has been observed in organic electronic devices ${ }^{7}$ and explored using theoretical simulations. ${ }^{8}$ Our recent work has elucidated the structure of the templating effect of highly crystalline films of CuI on both planar ${ }^{9}$ and non-planar MPc molecules.

The crystal structure of planar Pcs is inherently anisotropic due to face-to-face packing motifs leading to short intermolecular distances within stacks and larger inter-stack spacings. ${ }^{10}$ Structurally templating allows control of which of these spacings is predominantly observed in or out of the substrate plane. This in turn allows favorable charge transport pathways to be aligned in specific device architectures composed of crystalline thin films of MPc molecules.

In previous work on CuI templating we employed a continuous, highly crystalline, (111) oriented CuI layer and observed its influence on FePc layers grown at ambient substrate temperatures. Here we report the preparation and characterization of evaporated

\footnotetext{
${ }^{a}$ Department of Chemistry, The University of Warwick, Gibbet Hill Road, Coventry, CV4 7AL, UK.E-mail: l.rochford@warwick.ac.uk

${ }^{b}$ Department of Materials, Imperial College London, Exhibition Road, London, SW7 2AZ, UK
}

iron phthalocyanine (FePc)-CuI bilayer thin films at elevated substrate temperatures. The CuI films employed here are composed of isolated islands of $\mathrm{CuI}$ rather than continuous films, produced through deliberate micro-structuring induced by increasing substrate temperature during CuI growth. Crystallization sites in the early stages of FePc growth are observed at the edges of islands rather than on the top (111) faces. This leads to the growth of high aspect ratio needle-like crystallites in contrast to the continuous films previously observed in ambient temperature preparation of $\mathrm{FePc}$ on continuous $\mathrm{CuI}(111)$ films. ${ }^{9} \mathrm{XRD}$ patterns of the films produced using this methodology cannot be indexed using the single crystal structure of $\mathrm{FePc}$, and the presence of a new structural polymorph is suggested.

\section{Experimental details}

Copper iodide (Sigma Aldrich, UK) was used as received and evaporated from a home-built evaporator at $340{ }^{\circ} \mathrm{C}$ at a rate of $0.5 \AA^{-1}$ as measured by a calibrated quartz crystal microbalance. FePc (Sigma Aldrich, UK) was triply purified by thermal gradient sublimation and the resulting crystals were used for growth from a home-built evaporator at $375{ }^{\circ} \mathrm{C}$ at a rate of $0.2 \AA \mathrm{s}^{-1}$.

All films were grown in a custom-built ultra-high vacuum (UHV) chamber with a base pressure of $3 \times 10^{-9}$ mbar in which organic and inorganic materials were sublimed onto substrates held at elevated temperature. The substrate temperature was measured using a K-type thermocouple mounted close to the sample and calibrated using an optical pyrometer (IRCON ${ }^{\mathbb{R}}$ ). Substrates were $10 \times 10 \mathrm{~mm}$ pieces of thermally oxidised silicon (100) single crystal (IDB technologies, UK) cleaned in acetone, 
Decon- $90^{\circledR}$-deionised water mix and isopropanol. These were dried in a stream of dry nitrogen and UV-ozone cleaned before being loaded into vacuum.

Thin film XRD patterns were obtained using a PANalytical X'Pert Pro MRD diffractometer with monochromatic $\mathrm{Cu} \mathrm{K} \alpha_{1}$ radiation. Electron micrographs were recording using field emission scanning electron microscopy (FE-SEM, Zeiss Supra 55VP) with no additional film coating step. Atomic force microscopy (AFM) images were recorded using an Asylum research MFP-3D in AC mode (tapping mode) using Olympus AC240-TS silicon tips.

\section{Results}

High substrate temperatures are known to induce larger crystallite sizes in crystalline films of planar MPcs. ${ }^{11}$ To establish the behavior of FePc at high substrate temperatures, film growth was performed on clean $\mathrm{SiO}_{2}$ substrates. When $\mathrm{FePc}$ was grown at a substrate temperature of $180{ }^{\circ} \mathrm{C}$ to a thickness of $50 \mathrm{~nm}$ largely continuous films with interlocking grains were produced (Fig. 1). Angular holes and voids are observed as previously seen in high temperature growth on single crystal sapphire, ${ }^{12}$ and the structure is consistent with a highly crystalline (101) oriented (from comparison to CCDC no. 996880$)$ layer by XRD.

$\mathrm{CuI}$ films were then prepared for use as structural templating layers for high substrate temperature FePc film deposition. As previously reported, CuI thin films grow with their (111) plane oriented parallel to the substrate plane at ambient and elevated temperatures on $\mathrm{SiO}_{2}$ substrates. ${ }^{9}$ At high growth temperatures $\left(T_{\text {sub }}=180{ }^{\circ} \mathrm{C}\right), 15 \mathrm{~nm}$ thick films consist of isolated islands with predominantly hexagonal growth habits (Fig. 2). The height of these islands is up to $70 \mathrm{~nm}$ and a distribution of island heights is present with bare substrate between the crystallites. These micro-structured films were used at their growth temperature $\left(180{ }^{\circ} \mathrm{C}\right)$ as substrates for the subsequent growth of FePc films.

In $10 \mathrm{~nm}$ thick FePc films on CuI a sparse covering of high aspect ratio FePc needles (through which the underlying CuI(111) islands were visible) was observed (Fig. 3). A large spread in the lengths of these features was observed from SEM images and the largest dimension was approximately $1 \mu \mathrm{m}$. The shorter dimensions were more uniform with a value of approximately $50 \mathrm{~nm}$ for all of the needles present in this film. On closer inspection the distribution of these features across the surface was not random and seemed to be related to the morphology of the

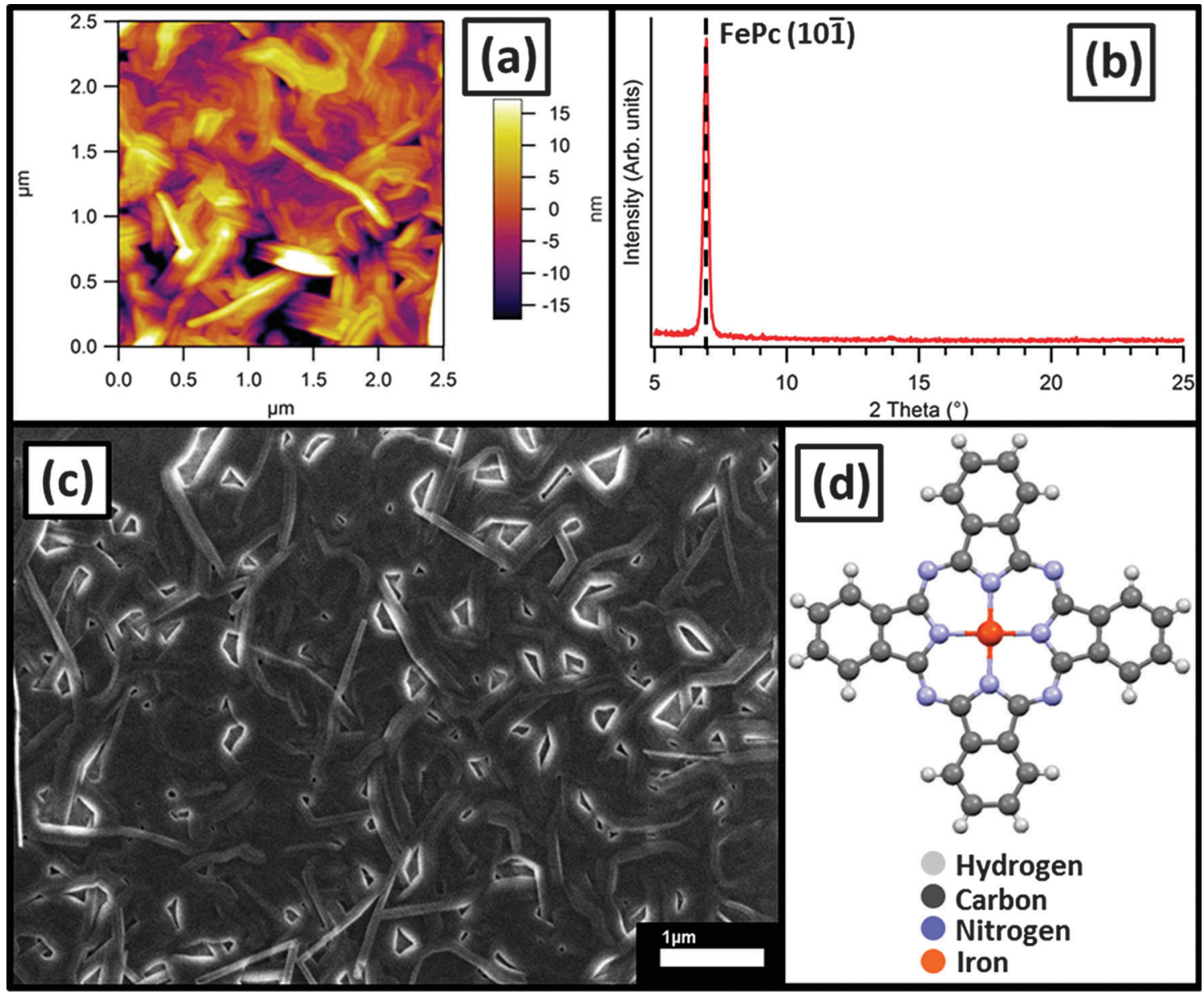

Fig. 1 (a) AFM topography image (b) XRD pattern and (c) SEM image of $50 \mathrm{~nm} \mathrm{FePc}-\mathrm{SiO}_{2}$ film grown at a substrate temperature of $180{ }^{\circ} \mathrm{C}$. A schematic of the FePc molecule is shown in (d). 


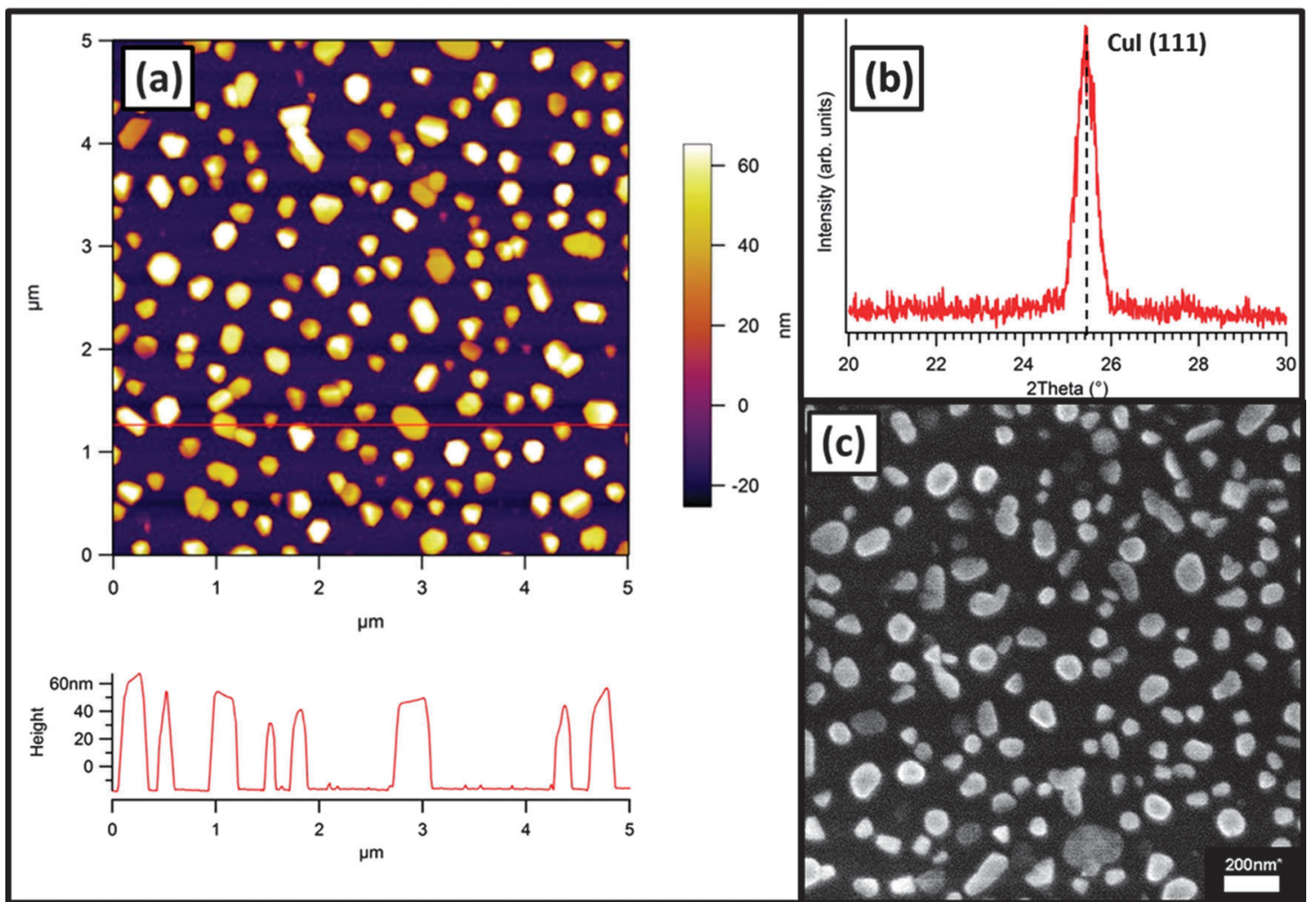

Fig. 2 (a) AFM image with corresponding cross-section (corresponds to line in image) (b) XRD pattern and (c) SEM image of $15 \mathrm{~nm} \mathrm{Cul}_{(111)}-\mathrm{SiO}{ }_{2}$ film grown at a substrate temperature of $180^{\circ} \mathrm{C}$.

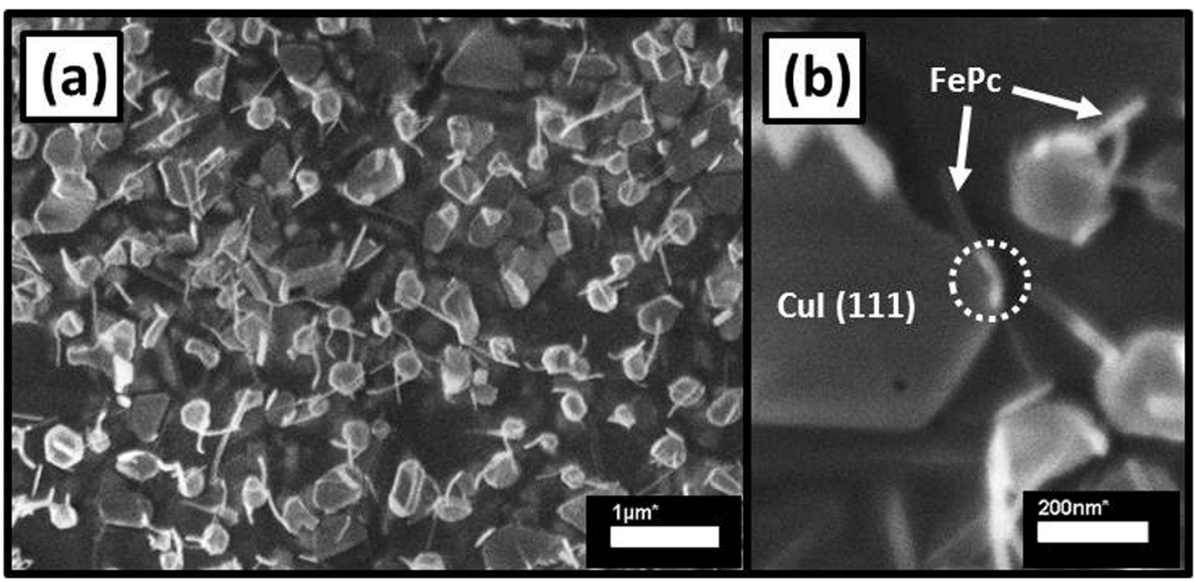

Fig. 3 (a) SEM image of $10 \mathrm{~nm} \mathrm{FePc}-\mathrm{Cul}(111)-\mathrm{SiO}_{2}$ film grown at $180{ }^{\circ} \mathrm{C}$ and (b) high-resolution SEM image of the same sample. Cul(111) and FePc Features are labelled in (b) and a nucleation site is highlighted with a white circle.

underlying CuI(111) islands. Crystals nucleated at the edges, rather than on the faces, of the $\mathrm{CuI}(111)$ islands and grew faster in one direction producing needle-like morphology; crystallites also seem to be protruding away from the substrate. Structural analysis by X-ray diffraction was attempted for this film but no peaks corresponding to FePc were observed due to the small amount of material (and therefore diffracting planes) present.

Thicker films were then grown $(50 \mathrm{~nm} \mathrm{FePc})$ to observe the evolution of the film morphology. The length of the needle-like
FePc crystals clearly increases with deposition time (longest observed dimension of approximately $4 \mu \mathrm{m}$ ) suggesting that they continue to grow throughout the deposition process rather than exclusively favoring nucleation of more crystallites (Fig. 4). A single growth direction is not evident as might be expected if the crystallites adopted a fixed orientation with respect to the CuI(111) faces. Areas with similar appearance to FePc films grown on bare $\mathrm{SiO}_{2}$ are also evident and exhibit the same angular voids as previously mentioned. This suggests that the 


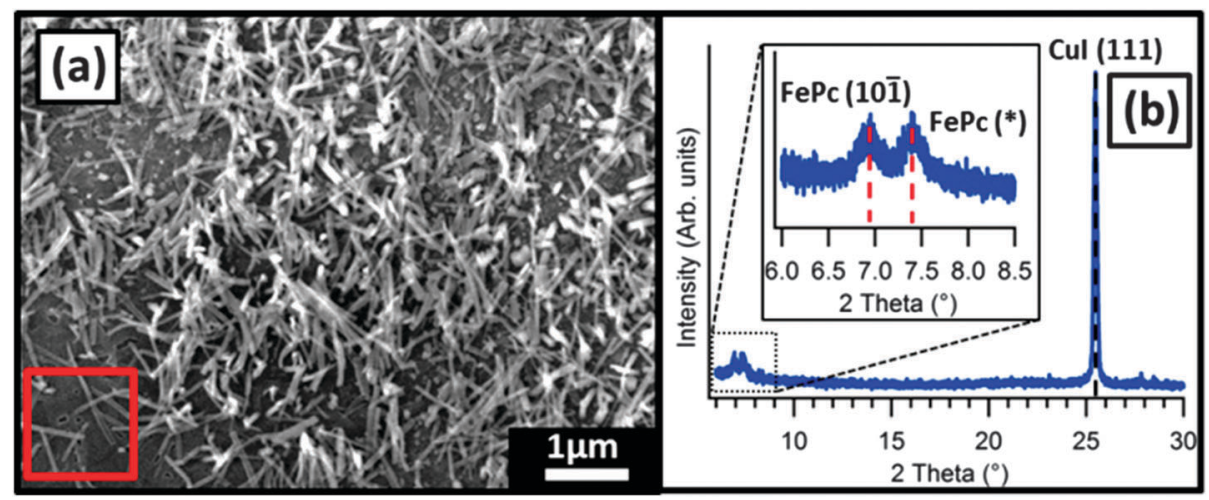

Fig. 4 (a) SEM image of $50 \mathrm{~nm} \mathrm{FePc}-\mathrm{Cul}(111)-\mathrm{SiO}_{2}$ film grown at $180{ }^{\circ} \mathrm{C}$ and (b) XRD pattern of the same sample with inset high-resolution scan. An area of growth of $\mathrm{FePc}$ on bare $\mathrm{SiO}_{2}$ is highlighted with a red box in (a).

growth of FePc still occurs in the voids between the CuI(111) islands (which are composed of clean $\mathrm{SiO}_{2}$ ) in the same (101) orientation already discussed.

\section{Discussion}

Previous studies have shown that when grown at ambient substrate temperature on a polycrystalline $\mathrm{CuI}(111)$ surface FePc molecules adopt a "lying down" orientation with the molecular plane parallel to the substrate surface. ${ }^{9}$ The $\operatorname{CuI}(111)$ surface studied here is different as the overall coverage of the surface by the overlayer is far lower, consisting of hexagonal crystallites surrounded by bare $\mathrm{SiO}_{2}$. As shown in SEM images, nucleation of FePc occurs at the edges of the CuI islands, which is not possible on complete CuI(111) films due to lack of exposed crystallite edges. The nature of these edges is therefore intrinsically important in the nucleation process of the FePc overlayer. Under ambient conditions CuI adopts a zinc blende face centred cubic (FCC) unit cell, as such the crystallite edges should correspond to the (100), (001) and (010) Miller planes. In this system the (100) and (001) planes are equivalent resulting in two possible orientations for the edges of the CuI crystallites, classified as $\{100\}$.

Planar MPcs have been shown to exhibit multiple polymorphs, the most common of those found in thin films typically termed $\alpha$ and $\beta$, with substrate temperatures below $200{ }^{\circ} \mathrm{C}$ leading to the $\alpha$-polymorph. ${ }^{13}$ Increasing growth temperature or post-growth annealing have been shown produce the $\beta$ polymorph. ${ }^{14}$ The tilt angle between MPc molecules is inherently different in the films composed of each polymorph. High temperature growth leads to a reduction of the distances between molecular columns, manifested in XRD patterns through equivalent peaks shifting to slightly higher angles than those of the $\alpha$-polymorph. The additional FePc peak present at $2 \theta=7.4^{\circ}\left(\mathrm{FePc}\left(^{*}\right)\right.$, Fig. $\left.4(\mathrm{~b})\right)$ in the high-temperature templated film, which cannot be interpreted with previously reported FePc structures (CCDC no. 996880 and Kirner et $a l .{ }^{15}$ ) and may therefore similarly be attributed to a new polymorph with reduced edge-to-edge intermolecular spacing. The central atom of the MPc influences the observed unit cell, but despite this the overall effects of the $\alpha$-to- $\beta$ transition upon XRD patterns are the same so comparison between planar phthalocyanines remains appropriate. Similar XRD patterns were previously observed in films of the $\beta$-polymorphs of the parent metal-free phthalocyanine $\left(\mathrm{H}_{2} \mathrm{Pc}\right)^{13}$ and copper(II) phthalocyanine $(\mathrm{CuPc}) .{ }^{16}$ While polymorphism in FePc has been suggested in the literature ${ }^{17}$ no structural data containing atomic positions suitable for XRD pattern simulation can account for diffraction at $2 \theta=7.4^{\circ}$. The change in peak position is, however, consistent with a polymorph transition in FePc although full structural analysis is not possible from such a small number of peaks.

\section{Conclusion}

Structural analysis suggests that the FePc grown on an incomplete CuI(111) layer consists of two distinct polymorphs of FePc. Edges of the CuI islands act as nucleation sites for the growth of high aspect ratio FePc crystallites which contain intermolecular spacings which cannot be indexed with a single FePc crystal structure. The change in $d$-spacing evident from the difference in peak position between the two FePc peaks is consistent with the presence of multiple polymorphs in other MPcs. The morphology of these crystallites is unlike that of FePc grown at high substrate temperatures on $\mathrm{SiO}_{2}$ and at ambient temperature on complete $\operatorname{CuI}(111)$ layers. This confirms that the combination of increased substrate temperature and the presence of the edges of $\mathrm{CuI}(111)$ islands is responsible for the growth of the polymorphic crystals.

\section{Author contributions}

The manuscript was written through contributions of all authors. All authors have given approval to the final version of the manuscript.

\section{Funding sources}

LAR and TSJ acknowledge support from the Engineering and Physical Sciences Research Council (EPSRC), UK (Grant no. EP/ H021388/1). AJR and SH thank the EPSRC for financial support via the Doctoral Training Centre in Plastic Electronics (Grant no. EP/G037515/1). 


\section{Acknowledgements}

LAR and TSJ acknowledge support from the Engineering and Physical Sciences Research Council, UK (Grant no. EP/H021388/1). AJR and SH thank the EPSRC for financial support via the Doctoral Training Centre in Plastic Electronics (Grant no. EP/G037515/1). The PANalytical MRD diffractometer used in this research was obtained through the Science City Advanced Materials Project: Creating and Characterizing Next Generation Advanced Materials, with support from Advantage West Midlands (AWM) and part funded by the European Regional Development Fund (ERDF).

\section{References}

1 S. Heutz, R. Cloots and T. S. Jones, Appl. Phys. Lett., 2000, 77, 3938.

2 P. Sullivan, T. S. Jones, A. J. Ferguson and S. Heutz, Appl. Phys. Lett., 2007, 91, 233114.

3 W. Wu, L. A. Rochford, S. Felton, Z. Wu, J. L. Yang, S. Heutz, G. Aeppli, T. S. Jones, N. M. Harrison and A. J. Fisher, J. Appl. Phys., 2013, 113, 013914.

4 Y. Zhou, T. Taima, T. Miyadera, T. Yamanari, M. Kitamura, K. Nakatsu and Y. Yoshida, Nano Lett., 2012, 12, 4146-4152. 5 S. R. Forrest, Chem. Rev., 1997, 97, 1793-1896.

6 C. H. Cheng, J. Wang, G. T. Du, S. H. Shi, Z. J. Du, Z. Q. Fan, J. M. Bian and M. S. Wang, Appl. Phys. Lett., 2010, 97, 083305.
7 T.-M. Kim, H. J. Kim, H.-S. Shim, M.-S. Choi, J. W. Kim and J.-J. Kim, J. Mater. Chem. A, 2014, 2, 8730.

8 B. P. Rand, D. Cheyns, K. Vasseur, N. C. Giebink, S. Mothy, Y. Yi, V. Coropceanu, D. Beljonne, J. Cornil, J.-L. Brédas and J. Genoe, Adv. Funct. Mater., 2012, 22, 2987-2995.

9 L. A. Rochford, D. S. Keeble, O. J. Holmes, G. J. Clarkson and T. S. Jones, J. Mater. Chem. C, 2014, 2, 6056.

10 A. Hoshino, Y. Takenaka and H. Miyaji, Acta Crystallogr., Sect. B: Struct. Sci., 2003, 59, 393-403.

11 J. E. S. Kim, E. Lim, K. Lee, D. Cha and B. Friedman, Appl. Surf. Sci., 2003, 205, 274-279.

12 C. Miller, A. Sharoni, G. Liu, C. Colesniuc, B. Fruhberger and I. Schuller, Phys. Rev. B: Condens. Matter Mater. Phys., 2005, 72, 104113.

13 S. M. Bayliss, S. Heutz, G. Rumbles and T. S. Jones, Phys. Chem. Chem. Phys., 1999, 1, 3673-3676.

14 S. Heutz, S. M. Bayliss, R. L. Middleton, G. Rumbles and T. S. Jones, J. Phys. Chem. B, 2000, 104, 7124-7129.

15 J. F. Kirner, W. Dow and W. R. Scheidt, Inorg. Chem., 1976, 15, 1685-1690.

16 S. Heutz, C. Mitra, W. Wu, A. J. Fisher, A. Kerridge, M. Stoneham, A. H. Harker, J. Gardener, H.-H. Tseng, T. S. Jones, C. Renner and G. Aeppli, Adv. Mater., 2007, 19, 3618-3622.

17 A. S. Milev, N. Tran, G. S. Kamali Kannangara, M. A. Wilson and I. Avramov, J. Phys. Chem. C, 2008, 112, 5339-5347. 